\title{
0 impacto da incorporação da inflação na análise de projetos de investimentos
}

\author{
Joana Siqueira de Souza ${ }^{a *}$, Francisco José Kliemann Neto ${ }^{b}$ \\ *ajoana.souza@pucrs.br, PUCRS, Brasil \\ bkliemann@producao.ufrgs.br, UFRGS, Brasil
}

\begin{abstract}
Resumo
Um processo de avaliação de investimentos realizado de forma satisfatória é essencial para que a empresa aplique seus recursos de capital em projetos corretos. Para isso, faz-se necessária a quantificação dos componentes do fluxo de caixa, bem como da depreciação e do impacto tributário do imposto de renda e da inflação nas análises econômico-financeiras. 0 objetivo deste trabalho é avaliar diferentes formas de incorporação da inflação nas avaliações, fazendo uma discussão sobre o papel da depreciação e seu impacto junto à inflação. 0 artigo aponta duas formas de incorporação da inflação: a forma homogênea, onde todos os componentes do fluxo de caixa são afetados pelo mesmo índice de inflação, e a forma heterogênea, onde cada componente é atualizado a partir de um índice diferente. Como resultado aponta-se o impacto desses diferentes conceitos nas análises, indicando a necessidade dos gestores atentarem para a questão da inflação nas análises de seus projetos.
\end{abstract}

Palavras-chave

Análise de investimentos. Depreciação. Inflação.

\section{Introdução}

A análise de investimentos tem se tornado cada vez mais importante, devido aos efeitos provocados por mudanças impostas pela atual conjuntura econômica, tais como políticas diferenciadas de suprimentos, forte competição internacional e o rápido avanço da tecnologia. Essas mudanças fazem com que os gerentes das empresas que almejam se manter competitivas no mercado trabalhem de forma sistêmica e dinâmica (ALBERTON et al., 2004).

No que tange ao processo de análise de investimentos alguns autores destacam o excesso de trabalhos que buscam discutir somente os métodos de avaliação de investimentos propriamente ditos, em detrimento de questões como a qualidade das informações consideradas nas análises (FARRAGHER; KLEIMAN; SAHU, 1999). Obviamente, a seleção de um adequado método de avaliação é essencial para o sucesso do processo de decisão, porém deve-se saber que o sucesso de uma análise depende, também, da melhoria do processo de investimento como um todo, considerando outras etapas anteriores e posteriores à avaliação, dentre elas a correta estimativa do fluxo de caixa econômico-financeiro do projeto (SOUZA, 2008).

Kliemann Neto (2006, p. 79) destaca que uma “[...] má análise de uma boa alternativa de investimento é melhor do que uma boa análise de uma má alternativa de investimento." 0 que o autor aponta é que, antes da discussão propriamente dita dos métodos de avaliação econômica de investimentos, deve-se procurar organizar o processo como um todo para permitir que as avaliações sejam feitas com dados coletados corretamente e com o envolvimento de pessoas preparadas. A partir disso, percebe-se uma necessidade de aprofundamento das discussões sobre alguns componentes que geram grande impacto no fluxo de caixa de projetos e, consequentemente, acarretam em grande impacto nas decisões finais das empresas: a incorporação da depreciação dos ativos e da inflação nas análises de investimento.

A depreciação pode ser contabilmente definida como uma despesa relativa à perda de valor de um determinado bem. Essa depreciação pode ser acarretada 
pela deterioração do bem ou pela obsolescência do mesmo, por exemplo (CASAROTTO FILHO; KOPITTKE, 2000). Existem alguns métodos para calcular o valor a ser depreciado do bem anualmente. 0 método mais utilizado e aceito pelo governo é o chamado linear, onde o valor de aquisição do bem é dividido pela sua vida útil contábil, conforme Equação 1.

$$
\text { Deprec }=\frac{P}{N}
$$

onde: Deprec $=$ valor a ser depreciado anualmente pela empresa; $P=$ preço de compra do bem; $N=$ vida útil do bem em anos.

Destaca-se que a depreciação não deve ser considerada um desembolso, pois o valor calculado não se classifica como uma saída de dinheiro efetiva. Porém, a depreciação é uma despesa, sendo assim passivel de ser abatida das receitas finais do período de trabalho da empresa, diminuindo, consequentemente, seu lucro tributável. Essa redução do lucro tributável da empresa acarretará também na redução do imposto de renda a ser pago, este por sua vez um desembolso real da empresa (CASAROTTO FILHO; KOPITTKE, 2000). Essas considerações têm impacto direto no fluxo de caixa de um investimento e, no contexto de avaliação de investimentos, devem ser levadas em consideração.

Para a definição da vida útil de determinado bem, a legislação fiscal adota certos parâmetros, restringindo a liberdade das empresas para a definição da taxa de depreciação. Isso é feito pois, em caso contrário, a tendência das empresas seria de depreciar o bem o mais rapidamente possível, para que o benefício fiscal se realize o quanto antes, como será discutido ao longo do artigo.

A questão da forma de incorporação da inflação também é um ponto importante quando se está avaliando investimentos. 0 objetivo principal de uma análise de investimentos é projetar um fluxo de caixa o mais próximo possível da realidade de sua implantação, para que o resultado desse investimento possa ser previamente quantificado, auxiliando na tomada de decisão (GALESNE; FENSTERSEIFER; LAMB, 1999). Dessa forma, percebe-se que a consideração da inflação nas análises de investimento é algo mandatório e, caso não adequadamente considerada, poderá ter impacto muito representativo no resultado final.

A partir disso, o objetivo principal deste artigo é discutir a importância da incorporação da depreciação e de inflação em análises de investimentos, buscando discutir as diferentes formas de incorporar tais parâmetros, fazendo uma análise crítica do impacto causado por elas. A seção seguinte apresentará uma discussão teórica, amparada por exemplos práticos.

\section{A depreciação e a inflação nas análises de investimentos}

0 fluxo de caixa do projeto é montado a partir dos dados de investimentos, receitas e custos do projeto. Ao reunir esses dados o avaliador terá a sua disposição o que se chama de Fluxo de Caixa Antes do Imposto de Renda (FCAl), ou seja, terá o resultado bruto do projeto, sem considerar demais encargos financeiros que deverão ocorrer ao implementar o projeto em questão. Entretanto, o impacto desses encargos é significativo, e deve ser levado em conta no momento da avaliação econômica de um projeto, buscando-se, assim, avaliar o Fluxo de Caixa Depois do Imposto de Renda (FCDI).

Para o cálculo do montante de imposto de renda e contribuição social a pagar, decorrente da execução de projeto, deve-se definir qual é o lucro tributável resultante em cada período de análise. Este lucro tributável é constituído do lucro líquido do projeto avaliado, subtraído do valor da depreciação dos investimentos realizados no projeto.

A depreciação dos investimentos é incorporada ao cálculo somente para reduzir o resultado dos períodos nos quais a mesma pode ser considerada. lsto é, se um determinado equipamento foi adquirido no período zero por um preço de $\mathrm{R} \$ 100.000,00$, e este é depreciado a uma taxa de $10 \%$ a.a., significa que poderá ser reduzido do resultado final do projeto $R \$ 10.000,00$ por ano, durante dez anos. Essa redução do resultado final terá um impacto direto no imposto de renda a pagar por parte da empresa, que se beneficiará com a redução do montante final de impostos a serem pagos. De acordo com Galesne, Fensterseifer e Lamb (1999, p. 107), o tratamento da depreciação dessa forma "[...] é efetivamente a regra utilizada pelas empresas quando do cálculo dos resultados." Segundo os autores, essa suposição parte do pressuposto que o déficit fiscal de um projeto implicará em realizar uma economia de imposto, que se realizará sobre o lucro de um outro projeto. Essa prática é chamada pelos autores de compensação interprojetos.

Suponha-se um projeto que exija um investimento de $\mathrm{R} \$ 9.000,00$ no período zero, que gere uma receita anual equivalente a $R \$ 13.000,00$, tenha custos anuais de manutenção equivalentes a $R \$ 4.000,00$, além de $R \$ 1.000,00$ anuais com mão de obra e $\mathrm{R} \$ 3.000,00$ anuais com materiais. Suponha-se ainda que esse projeto tenha uma vida útil de três anos, na qual o investimento inicial poderá ser totalmente depreciado. Considere-se, ainda, que a taxa mínima de atratividade (TMA) da empresa é de 10\% ao ano e que a taxa de imposto de renda mais contribuição social seja de 34\% ao ano.

Para avaliar esse projeto métodos tradicionais de avaliação de investimentos serão empregados, entre 
eles o valor presente líquido (VPL) e o método da taxa interna de retorno (TIR).

Montando o fluxo de caixa para este projeto (Tabela 1), pode-se perceber o impacto que o pagamento do imposto de renda e da contribuição social acarreta no resultado final do projeto. Esse investimento, que antes apresentava um resultado bastante interessante para a empresa, quando avaliado após o pagamento dos tributos apresenta um resultado ainda satisfatório, porém bem abaixo daquele encontrado anteriromente.

Entretanto, apenas a incorporação do impacto da depreciação e do pagamento de tributos torna-se insuficiente para que a análise de investimentos seja feita de forma correta, apresentando um resultado aderente à realidade da empresa. Para que a análise possa simular situações futuras, faz-se necessário que a inflação dos períodos analisados seja levada em consideração na montagem do fluxo de caixa do projeto avaliado.

Casarotto Filho e Kopittke (2000) definem a inflação como sendo a perda do poder aquisitivo da moeda, provocada pelo aumento diferenciado do preço de produtos e serviços, que acaba acarretando na diminuição do valor real da moeda, por exemplo. Sendo assim, como essa variação pode influenciar diretamente nos cálculos de engenharia econômica durante a análise de investimentos, faz-se necessário que a inflação seja considerada quando a mesma ocorrer.

\subsection{Análise considerando-se inflação homogênea}

Se todos os elementos que constituem o fluxo de caixa do projeto que está sendo avaliado são afetados da mesma forma pela inflação, esta pode ser denominada inflação homogênea. Ou seja, a inflação homogênea é aquela onde todos os itens de receitas e custos são inflacionados à mesma taxa, sendo todo o fluxo então inflacionado à mesma taxa de inflação. Quando isso ocorre, deve-se inflacionar também a TMA da empresa, utilizando para o cálculo do VPL a chamada taxa nominal, conforme mostra a Equação 2 (SMART; MEGGINSON; GITMAN, 2004).

$\left(1+i_{n}\right)=(1+T M A) \times\left(1+i_{\text {inf }}\right)$

onde: $i_{n}$ taxa nominal; TMA = TMA real da empresa; $i_{\text {inf }}=$ taxa da inflação do período.

Retomando o exemplo do projeto anteriormente avaliado, deve-se incorporar ao fluxo de caixa a inflação estimada para cada período de análise. Caso a empresa opte por trabalhar com a inflação homogênea, então a taxa de inflação dos períodos deve ser estimada, e a partir desta taxa deve ser calculada a taxa nominal. Após, todos os componentes do fluxo de caixa devem ser inflacionados à taxa de inflação definida.

Para o exemplo anterior, a empresa definiu uma taxa de inflação homogênea de 5,5\% ao ano. Dessa forma, a taxa nominal a ser utilizada é de 16,05\% ao ano, conforme mostra a Tabela 2.

Pode-se observar nas Tabelas 1 e 2 que o VPL do projeto antes do impacto do pagamento de imposto de renda e contribuição social não se altera com a incorporação da inflação homogênea. Entretanto, observa-se que ao indicador do método da TIR apresenta modificação aparente passando de 30,64\% ao ano, no projeto sem a consideração da inflação, para 37,82\% ao ano, após a incorporação da taxa de inflação. Porém, esses dois índices não podem ser diretamente confrontados, pois o segundo apresenta agregado à sua taxa anual uma taxa inflação

Tabela 1. Fluxo de caixa para o projeto analisado, sem consideração da inflação.

\begin{tabular}{|c|c|c|c|c|c|c|c|c|c|}
\hline Ano & Investimento & Receitas & Manutenção & Mão de obra & Materiais & FCAl & Depreciação & IR & FCDI \\
\hline 0 & $(9.000,00)$ & - & - & - & - & $(9.000,00)$ & - & - & $(9.000,00)$ \\
\hline 1 & - & $13.000,00$ & $4.000,00$ & $1.000,00$ & $3.000,00$ & $5.000,00$ & $3.000,00$ & 680,00 & $4.320,00$ \\
\hline 2 & - & $13.000,00$ & $4.000,00$ & $1.000,00$ & $3.000,00$ & $5.000,00$ & $3.000,00$ & 680,00 & $4.320,00$ \\
\hline 3 & - & $13.000,00$ & $4.000,00$ & $1.000,00$ & $3.000,00$ & $5.000,00$ & $3.000,00$ & 680,00 & $4.320,00$ \\
\hline \multirow{2}{*}{\multicolumn{2}{|c|}{ TMA REAL $=10,00 \%$}} & & & & VPL & $3.434,26$ & & VPL & $1.743,20$ \\
\hline & & & & & TIR & $30,64 \%$ & & $\mathrm{TIR}$ & $20,71 \%$ \\
\hline
\end{tabular}

Tabela 2. Fluxo de caixa para o projeto avaliado, considerando inflação homogênea.

\begin{tabular}{|c|c|c|c|c|c|c|c|c|c|}
\hline Ano & Investimento & Receitas & Manutenção & Mão de obra & Materiais & FCAl & Depreciação & IR & FCDI \\
\hline 0 & $(9.000,00)$ & - & - & - & - & $(9.000,00)$ & - & - & $(9.000,00)$ \\
\hline 1 & - & $13.715,00$ & $4.220,00$ & $1.055,00$ & $3.165,00$ & $5.275,00$ & $3.000,00$ & 773,50 & $4.501,50$ \\
\hline 2 & - & $14.469,33$ & $4.452,10$ & $1.113,03$ & $3.339,08$ & $5.565,13$ & $3.000,00$ & 872,14 & $4.692,98$ \\
\hline 3 & - & $15.265,14$ & $4.696,97$ & $1.174,24$ & $3.522,72$ & $5.871,21$ & $3.000,00$ & 976,21 & $4.895,00$ \\
\hline \multirow{3}{*}{\multicolumn{3}{|c|}{ TMA NOMINAL $1=16,05 \%$}} & & & VPL & $3.434,26$ & & VPL & $1.495,54$ \\
\hline & & & & & $\mathrm{TIR}$ & $37,82 \%$ & & $\mathrm{TIR}$ & $25,82 \%$ \\
\hline & & & & & TIR LIQ & $30,64 \%$ & & TIR LIQ & $19,26 \%$ \\
\hline
\end{tabular}


homogênea estimada em 5,5\% ao ano. Dessa forma, para que se torne comparável, deve-se utilizar como indicador a TIR líquida (TIR LIQ), que representa a taxa de retorno real, sem o valor da inflação estimada anteriormente. Nesse caso, a TIR líquida do projeto antes do pagamento dos impostos mostra-se constante independente da incorporação ou não de uma taxa de inflação homogênea.

Percebe-se também, a partir dos resultados econômicos das Tabelas 1 e 2, que o projeto que antes apresentava um VPL de R $\$ 1.743,20$ após o pagamento de impostos e sem a consideração da inflação, agora apresenta um resultado de $R \$ 1.495,54$. Observando somente a Tabela 2, percebe-se que a depreciação foi o único componente do fluxo de caixa que não foi inflacionado, fazendo-se necessária uma discussão sobre essa premissa. No Brasil, a legislação tributária (artigo 186 do Decreto $\mathrm{n}^{\circ}$ 58.400, de 10 de maio de 1966) permite que a depreciação seja computada como despesa a fim de dedução do imposto de renda, mas ela não pode ser atualizada de acordo com nenhum indice monetário. Dessa forma, o valor da depreciação não pode ser inflacionado, dado que o mesmo não poderá ser corrigido com o passar dos anos.

Observando novamente as Tabelas 1 e 2 pode-se perceber que o resultado do VPL antes da aplicação da taxa de imposto de renda e contribuição social são iguais, isto é, a consideração de inflação homogênea no fluxo de caixa não afeta o resultado final do projeto. A diferença nos valores identificada no resultado após o imposto decorre, assim, da não atualização da depreciação. Ou seja, o fato da depreciação não ser atualizável é que causa a distorção no resultado final, dado que a empresa abaterá no imposto uma parcela da depreciação que anualmente seria atualizada à taxa de $0 \%$, enquanto os outros itens do fluxo de caixa seriam atualizados a uma taxa de inflação, que nesse exemplo é de 5,5\% ao ano.

A Equação 3 apresenta, então, uma maneira genérica de calcular o VPL de um projeto com a consideração de um índice de inflação. Percebe-se que a depreciação não pode ser inflacionada, enquanto todos os outros componentes do fluxo de caixa devem ser inflacionados à taxa de inflação definida para os períodos de análise.

$$
\begin{aligned}
V P L= & F C_{0}+\sum_{n=1}^{t}\left[\frac{F C_{n} \times\left(1+i_{I N F L}\right)^{n}}{(1+T M A)^{n} \times\left(1+i_{I N F L}\right)^{n}}-\right. \\
& \left.\left(\frac{F C_{n} \times\left(1+i_{I N F L}\right)^{n}-\text { Deprec }}{(1+T M A)^{n} \times\left(1+i_{I N F L}\right)^{n}}\right) \times I R\right]
\end{aligned}
$$

onde: $F C_{o}=$ investimento inicial; $F C_{n}=$ fluxo de caixa do projeto no período $n$ (receitas - custos); $i_{\text {INFL }}=$ taxa de inflação estimada para o período $n$; $T M A=$ taxa mínima de atratividade da empresa; Deprec $=$ depreciação do período; $I R=$ taxa do imposto de renda e contribuição social; $n=$ período do fluxo de caixa $(1,2,3, \ldots, t)$.

Observando a Equação 3, percebe-se que é possível simplificá-la cortando os numeradores e denominadores em comum, resultando na Equação 4.

$$
\begin{aligned}
V P L & =F C_{0}+\sum_{n=1}^{t}\left[\frac{F C_{n}}{(1+T M A)^{n}}\right. \\
& \left.-I R \times\left(\frac{F C_{n}}{(1+T M A)^{n}}-\frac{\text { Deprec }}{(1+T M A)^{n} \times\left(1+i_{I N F L}\right)^{n}}\right)\right]
\end{aligned}
$$

Ou seja, outra forma de incorporar a inflação homogênea na avaliação econômica de um projeto é a partir da descapitalização da depreciação, uma vez que esta, conforme a legislação, não pode ser atualizada ou reajustada de acordo com a inflação do período. Sendo assim, como todos os fluxos de caixa devem ser inflacionados de acordo com a inflação do período e, posteriormente, ao calcular-se o VPL, são deflacionados à mesma taxa de inflação, percebe-se que o efeito diferenciado da inflação se dará na depreciação.

Avaliando novamente o projeto exemplificado nas Tabelas 1 e 2, utilizando desta vez a Equação 4, onde os fluxos de caixa permanecem constantes, exceto o valor da depreciação que deve ser descapitalizado, deve-se chegar ao mesmo resultado econômico encontrado na Tabela 2, conforme mostra a Tabela 3.

Observando o resultado do VPL nas Tabelas 2 e 3 percebe-se que as duas formas de incorporar a

\begin{tabular}{|c|c|c|c|c|c|c|c|c|c|}
\hline Ano & Investimento & Receitas & Manutenção & Mão de obra & Materiais & FCAl & Depreciação & IR & FCDI \\
\hline 0 & $(9.000,00)$ & - & - & - & - & $(9.000,00)$ & - & - & $(9.000,00)$ \\
\hline 1 & - & $13.000,00$ & $4.000,00$ & $1.000,00$ & $3.000,00$ & $5.000,00$ & $2.843,60$ & 733,18 & $4.266,82$ \\
\hline 2 & - & $13.000,00$ & $4.000,00$ & $1.000,00$ & $3.000,00$ & $5.000,00$ & $2.695,36$ & 783,58 & $4.216,42$ \\
\hline 3 & - & $13.000,00$ & $4.000,00$ & $1.000,00$ & $3.000,00$ & $5.000,00$ & $2.554,84$ & 831,35 & $4.168,65$ \\
\hline \multirow{2}{*}{\multicolumn{2}{|c|}{ TMA REAL $=10,00 \%$}} & & & & VPL & $\mathrm{R} \$ 3.434,26$ & & VPL & $\mathrm{R} \$ 1.495,54$ \\
\hline & & & & & TIR & $30,64 \%$ & & TIR & $19,26 \%$ \\
\hline
\end{tabular}
inflação homogênea são passíveis de serem realizadas apresentando o mesmo resultado. Destaca-se que

Tabela 3. Fluxo de caixa do projeto analisado, considerando inflação homogênea, com descapitalização da depreciação. 
a maneira mais simples de considerar a inflação na análise é justamente ignorando-a no fluxo de caixa. lsso porque a inflação nesse caso irá atingir os elementos do fluxo de caixa de forma equivalente, não afetando o valor final da análise. Quando a situação é essa, a taxa de desconto utilizada deve ser a TMA real definida pela empresa (GALESNE; FENSTERSEIFER; LAMB, 1999).

Além disso, pode-se observar que a depreciação, por não ser atualizável perante a lei, trata-se de um elemento do fluxo de caixa que não sofre uma inflação equivalente à dos demais elementos, mesmo em um ambiente onde se considera inflação homogênea. lsso leva à conclusão de que quando considerada a depreciação em uma análise de investimentos, esta deve ser incorporada de modo que sofra reajuste de 0\% ao ano.

\subsection{Análise considerando inflação heterogênea}

As discussões realizadas até agora consideram que todos os componentes dos fluxos de caixa serão afetados pelo mesmo índice de inflação, o que algumas vezes pode não representar a realidade enfrentada por muitas empresas.

Quando os elementos constituintes do fluxo de caixa são afetados de forma diferenciada pela inflação, isto é, alguns itens variam mais e outros menos, há a necessidade de se considerar essas diferenças na avaliação econômica. Nesse caso, cada componente do fluxo de caixa deve ser inflacionado à sua taxa específica, sendo calculada então a taxa média de inflação do período, que será uma média ponderada dos diferentes índices de inflação, conforme a equação (5). Com essa taxa média de inflação será possivel calcular a taxa nominal da empresa, que servirá para calcular o resultado econômico do projeto.

$$
\begin{array}{r}
i_{I N F M E D I A}=\frac{\left[\left(C_{1} \times i_{I N F 1}\right)+\left(C_{2} \times i_{I N F 2}\right)\right]}{C_{1}+C_{2}+R_{1}+\ldots+R_{n}}+ \\
\frac{\left[\left(R_{1} \times i_{I N F 3}\right)+\ldots+\left(R_{n} \times i_{I N F t}\right)\right]}{C_{1}+C_{2}+R_{1}+\ldots+R_{n}}
\end{array}
$$

onde: $C_{n}$ e $R_{n}=$ componentes do fluxo de caixa do projeto avaliado; $i_{I N F t}=$ índices de inflação de cada componente do fluxo de caixa.

Importante salientar que a consideração dessa formulação permitirá que a empresa determine uma taxa de inflação média e interna às suas atividades, diferente de valores de inflação médios publicados e externos à empresa.

Retomando o exemplo do projeto avaliado anteriormente, caso a empresa deseje incorporar à análise diferentes índices de inflação para cada componente do fluxo de caixa, a mesma deve inflacionar cada um dos componentes do fluxo de acordo com esses índices, e logo após, para calcular o VPL do projeto, deve-se calcular a taxa de inflação média do período e a taxa nominal da empresa.

Considere-se que a empresa estime uma inflação de $2 \%$ ao ano para suas receitas, um aumento de 5,5\% nos custos de manutenção, de 4\% na mão de obra e um aumento considerável de 10,5\% ao ano nos seus custos com materiais, devido ao aumento nos custos de algumas matérias-primas.

A inflação média considerada em cada período foi calculada a partir da Equação 5, conforme segue.

$$
\begin{aligned}
i_{\text {INFMÉDIA }}=\frac{[(13.000 \times 2 \%)+(4.000 \times 5,5 \%)]}{13.000+4.000+1.000+3.000}+ \\
\frac{[(1.000 \times 4 \%)+(3.000 \times 10,5 \%)]}{13.000+4.000+1.000+3.000} \\
i_{\text {INFMÉDIA }}=3,98 \% \text { a.a. }
\end{aligned}
$$

A partir dessa taxa média de inflação e da TMA da empresa foi possível calcular a TMA nominal, demonstrada a seguir.

$\left(1+i_{n}\right)=(1+10 \%) \times(1+3,98 \%)$

$i_{n}=14,37 \%$ a.a.

De posse dessa TMA nominal, recalcula-se os resultados do projeto, observando-se uma queda significativa nos resultados, conforme Tabela 4.

A incorporação de uma inflação heterogênea,

\begin{tabular}{|c|c|c|c|c|c|c|c|c|c|}
\hline Ano & Investimento & Receitas & Manutenção & Mão de obra & Materiais & FCAl & Depreciação & IR & FCDI \\
\hline 0 & $(9.000,00)$ & - & - & - & - & $(9.000,00)$ & - & - & $(9.000,00)$ \\
\hline 1 & - & $13.260,00$ & $4.220,00$ & $1.040,00$ & $3.315,00$ & $4.685,00$ & $3.000,00$ & 572,90 & $4.112,10$ \\
\hline 2 & - & $13.525,20$ & $4.452,10$ & $1.081,60$ & $3.663,08$ & $4.328,43$ & $3.000,00$ & 451,66 & $3.876,76$ \\
\hline 3 & - & $13.795,70$ & $4.696,97$ & $1.124,86$ & $4.047,70$ & $3.926,18$ & $3.000,00$ & 314,90 & $3.611,28$ \\
\hline & & & & & VPL & $1.029,22$ & & VPL & $-27,42$ \\
\hline \multirow{2}{*}{\multicolumn{3}{|c|}{ TMA NOMINAL $2=14,37 \%$}} & & & $\mathrm{TIR}$ & $21,33 \%$ & & TIR & $14,19 \%$ \\
\hline & & & & & TIR LIQ & $16,69 \%$ & & TIR LIQ & $9,82 \%$ \\
\hline
\end{tabular}
onde cada componente do fluxo de caixa é afetado por uma inflação específica, torna-se mais complexa, uma vez que cada dono de projeto deve buscar uma inflação para cada tipo de componente do seu fluxo e

Tabela 4. Fluxo de caixa do projeto analisado, considerando inflação heterogênea. 
calcular uma taxa nominal ponderada de desconto a ser utilizada para determinar o resultado econômico de seu projeto. Entretanto, muitas vezes essa sofisticação na análise se faz necessária para que a mesma seja aderente à realidade do projeto.

No exemplo apresentado nas Tabelas 1, 2, 3 e 4 percebe-se que o projeto apresenta resultado econômico menor à medida que a inflação é incorporada. No caso da consideração de inflação heterogênea, o projeto que antes apresentava VPL igual a R\$1.495,54 e TIR LIQ igual a 19,26\% a.a., tornou-se um projeto inviável com VPL igual a $\mathrm{R} \$-27,42$ e TIR LIQ de 9,82\% a.a. Ou seja, o fato da inflação que atinge os custos do projeto ser maior que a inflação que atinge as receitas faz com que o resultado do projeto seja reduzido, tornando-se menos atrativo para a empresa. Porém, se os índices de inflação estão bem estimados, a consideração da inflação heterogênea apresenta resultados bem mais condizentes com a realidade que a empresa enfrentará, reduzindo a probabilidade de erro nas análises ou a escolha de projetos de forma equivocada.

Destaca-se que caso a inflação das receitas fosse maior que a inflação projetada para os itens de custos, o resultado do projeto seria melhor, indicando um determinado crescimento das receitas frente aos custos. Da mesma forma, a utilização de inflação heterogênea permitirá uma melhor análise do futuro que a empresa enfrentará, apontando um cenário com maior probabilidade de ocorrência.

No exemplo aqui demonstrado, há um pressuposto implícito de que os índices de inflação heterogênea se manterão constantes por elemento analisado ao longo do período de análise do projeto, isto é, as eventuais diferenças entre os índices são potencializados pelo tempo. Uma análise mais profunda exigiria a definição de índices de inflação diferenciados por elemento do fluxo de caixa e por período de análise. Além disso, a inflação média deveria ser recalculada para cada um dos períodos, pois seu peso relativo se altera em decorrência das variações diferenciadas proporcionadas pela inflação heterogênea. Essas são possíveis melhorias quando da consideração de inflações heterogêneas no fluxo de caixa que só fariam aumentar a qualidade da análise, porém que também devem ser questionadas quanto ao benefício agregado frente à sofisticação no cálculo.

\subsection{Análise do impacto do prazo de depreciação}

Outro ponto de destaque que pode ser observado nas Tabelas 1, 2, 3 e 4 é a questão do prazo de depreciação permitido pela legislação. Anteriormente discutiu-se que a vida útil dos bens da empresa pode ser definida pela empresa, dentro dos limites impostos pelo governo. lsso porque, caso o período ficasse a cargo das empresas, estas depreciariam seus bens o quanto antes para beneficiar-se dos efeitos fiscais também o mais cedo possível. Com a restrição de não poder atualizar a depreciação ao longo dos períodos e com o impacto da desvalorização da moeda período a período o impacto do prazo de depreciação torna-se ainda maior.

Retomando o exemplo do projeto em análise, considerando que a empresa possa depreciar seu investimento em dois anos, e não mais em três, como havia sido estimado, as Tabelas 5 e 6 apresentam

Tabela 5. Fluxo de caixa do projeto analisado, considerando depreciação antecipada e inflação homogênea.

\begin{tabular}{|c|c|c|c|c|c|c|c|c|c|}
\hline Ano & Investimento & Receitas & Manutenção & Mão de obra & Materiais & FCAl & Depreciação & IR & FCDI \\
\hline 0 & $(9.000,00)$ & - & - & - & - & $(9.000,00)$ & - & - & $(9.000,00)$ \\
\hline 1 & - & $13.715,00$ & $4.220,00$ & $1.055,00$ & $3.165,00$ & $5.275,00$ & $4.500,00$ & 263,50 & $5.011,50$ \\
\hline 2 & - & $14.469,33$ & $4.452,10$ & $1.113,03$ & $3.339,08$ & $5.565,13$ & $4.500,00$ & 362,14 & $5.202,98$ \\
\hline 3 & - & $15.265,14$ & $4.696,97$ & $1.174,24$ & $3.522,72$ & $5.871,21$ & & $1.996,21$ & $3.875,00$ \\
\hline \multirow{3}{*}{\multicolumn{3}{|c|}{ TMA NOMINAL $1=16,05 \%$}} & & & VPL & $3.434,26$ & & VPL & $1.661,07$ \\
\hline & & & & & TIR & $37,82 \%$ & & $\mathrm{TIR}$ & $27,51 \%$ \\
\hline & & & & & TIR LIQ & $30,64 \%$ & & TIR LIQ & $20,86 \%$ \\
\hline
\end{tabular}

Tabela 6. Fluxo de caixa do projeto analisado, considerando depreciação antecipada e inflação heterogênea.

\begin{tabular}{|c|c|c|c|c|c|c|c|c|c|}
\hline Ano & Investimento & Receitas & Manutenção & Mão de obra & Materiais & FCAl & Depreciação & IR & FCDI \\
\hline 0 & $(9.000,00)$ & - & - & - & - & $(9.000,00)$ & - & - & $(9.000,00)$ \\
\hline 1 & - & $13.260,00$ & $4.220,00$ & $1.040,00$ & $3.315,00$ & $4.685,00$ & $4.500,00$ & 62,90 & $4.622,10$ \\
\hline 2 & - & $13.525,20$ & $4.452,10$ & $1.081,60$ & $3.663,08$ & $4.328,43$ & $4.500,00$ & $(58,34)$ & $4.386,76$ \\
\hline 3 & - & $13.795,70$ & $4.696,97$ & $1.124,86$ & $4.047,70$ & $3.926,18$ & - & $1.334,90$ & $2.591,28$ \\
\hline & & & & & VPL & $1.029,22$ & & VPL & 126,61 \\
\hline \multirow{2}{*}{\multicolumn{3}{|c|}{ TMA NOMINAL $2=14,37 \%$}} & & & TIR & $21,33 \%$ & & $\mathrm{TIR}$ & $15,29 \%$ \\
\hline & & & & & TIR LIQ & $16,69 \%$ & & TIR LIQ & $10,88 \%$ \\
\hline
\end{tabular}


o novo resultado do projeto caso a empresa utilize a inflação homogênea (Tabela 5) ou heterogênea (Tabela 6).

Percebe-se que, no caso do projeto com inflação homogênea, o resultado considerando a depreciação do investimento em três anos era VPL $=\mathrm{R} \$ 1.495,54$. Com a antecipação da depreciação o projeto passou a apresentar VPL $=\mathrm{R} \$ 1.661,07$, ou seja, o resultado para a empresa aumentou. No projeto com inflação diferenciada o resultado foi semelhante, antes o projeto apresentava $\mathrm{VPL}=\mathrm{R} \$-27,42$, e com o prazo de depreciação diminuído apresentou VPL $=\mathrm{R} \$ 126,61$, ou seja, o projeto tornou-se viável.

Este exemplo prova que quanto antes a empresa conseguir depreciar seus investimentos, melhor será o resultado econômico-financeiro da organização, principalmente devido à redução do impacto da desvalorização da moeda na estrutura de capital da empresa.

\subsection{Análise do impacto do valor residual}

Outro ponto que deve ser discutido quando se fala do impacto da inflação nas análises é a consideração do valor residual dos bens adquiridos para a execução do projeto. Quando a venda de um equipamento ocorre ao final de sua vida útil contábil, o valor residual considerado na análise é o valor do bem totalmente depreciado, incidindo o imposto de renda sobre todo o valor de venda declarado.

Caso a venda do bem ocorra antes do último período da vida útil contábil do equipamento, ou seja, antes da depreciação contábil completa do bem, deve constar nos cálculos um outro item chamado "ônus do imposto de renda", isto é, o valor que a empresa deve pagar de impostos sobre a venda final do bem, descontando a parte até então não depreciada (valor contábil), conforme mostra a Equação 6.

Ônus do $\mathbb{I R}_{n}=\left(\right.$ valor residual - valor contábil $\left.{ }_{n}\right) \times \mathbb{I R}$

onde: Ônus do $\mathrm{IR}=$ valor a ser pago de impostos referente à venda do bem; Valor residual = valor de revenda do bem; $\mathrm{IR}=$ taxa do imposto de renda; $n=$ número de períodos em que o bem foi usado.
0 valor contábil do bem representa o quanto ele ainda vale, sob o ponto de vista contábil. Dessa forma, esse valor é representado pelo valor de compra do bem subtraído do valor que já fora depreciado do mesmo, conforme mostra a Equação 7.

Valor contábil ${ }_{n}=$ Compra $-\sum_{i=1}^{n}$ Deprec

onde: Valor contábil ${ }_{n}=$ valor do bem, sob o ponto de vista contábil; Compra $=$ valor de compra do bem; $\sum_{i=1}^{n}$ Deprec $=$ somatório do valor depreciado até o período $n$.

Tanto o valor de compra quanto os valores depreciados até então são valores não atualizáveis, o que faz com que os mesmos não possam ser inflacionados no fluxo de caixa, permanecendo constantes. Caso a empresa opte pela incorporação da inflação homogênea no fluxo de caixa a partir da descapitalização da depreciação, conforme a Equação 4, então o valor contábil também deverá ser descapitalizado à mesma taxa de inflação definida para os períodos conforme a Equação 8.

Valor contábil ${ }_{n}=\frac{\text { Compra }-\sum_{i=1}^{n} \text { Deprec }}{\left(1+i_{I N F}\right)^{n}}$

Retomando o exemplo utilizado, imagine-se que a empresa resolva vender o equipamento comprado com o investimento inicial de $\mathrm{R} \$ 9.000,00$ ao final do segundo ano de uso do bem. 0 valor residual estimado para o ativo é de $\mathrm{R} \$ 4.000,00$. Dessa forma, além da taxa de imposto de renda e contribuição social que a empresa deverá desembolsar ao final do segundo ano equivalente a $\mathrm{R} \$ 783,58$, a empresa deverá pagar um ônus de imposto sobre a venda do bem acima do valor contábil, estabelecido em R\$3.000,00, conforme segue.

$$
\begin{aligned}
\text { Ônus do IR2 } & =34 \% \times[4.000,00 \\
& \left.-\frac{9.000,00-6.000,00}{(1+5,5 \%)^{2}}\right]=443,58
\end{aligned}
$$

Na Tabela 7, pode-se observar todo o fluxo de caixa, considerando a venda de bem no período

\begin{tabular}{|c|c|c|c|c|c|c|c|c|c|c|c|}
\hline Ano & Investimento & Receitas & Manutenção & $\begin{array}{c}\text { Mão de } \\
\text { obra }\end{array}$ & Materiais & VR & FCAl & Depreciação & IR & $\begin{array}{l}\text { Ônus } \\
\text { do IR }\end{array}$ & FCDI \\
\hline 0 & $(9.000,00)$ & - & - & - & - & - & $(9.000,00)$ & - & - & - & $(9.000,00)$ \\
\hline 1 & - & $13.000,00$ & $4.000,00$ & $1.000,00$ & $3.000,00$ & - & $5.000,00$ & $2.843,60$ & 733,18 & - & $4.266,82$ \\
\hline 2 & - & $13.000,00$ & $4.000,00$ & $1.000,00$ & $3.000,00$ & $4.000,00$ & $9.000,00$ & $2.695,36$ & 783,58 & 443,58 & $7.772,84$ \\
\hline \multirow{2}{*}{\multicolumn{2}{|c|}{ TMA REAL $=10,00 \%$}} & & & & & VPL & $\begin{array}{c}\mathrm{R} \$ \\
2.983,47\end{array}$ & & & VPL & $\begin{array}{c}\mathrm{R} \$ \\
1.302,77\end{array}$ \\
\hline & & & & & & TIR & $31,56 \%$ & & & TIR & $19,61 \%$ \\
\hline
\end{tabular}

Tabela 7. Fluxo de caixa do projeto analisado, considerando a venda do bem ao final do segundo ano e inflação homogênea. 
anteriormente definido. $0 \mathrm{VPL}$ do projeto considerando a depreciação contábil completa do equipamento, sem valor residual, é equivalente a $R \$ 1.495,54$. Caso a empresa resolva desativar o projeto ao final do segundo ano, vendendo o ativo por $\mathrm{R} \$ 4.000,00$, o VPL do projeto resulta em $\mathrm{R} \$ 1.302,77$, mostrando ser uma decisão que retorna um valor inferior à empresa.

\section{Conclusões}

Torna-se claro para um gestor, quando da avaliação de seus investimentos, que a inflação que impactará sobre o fluxo de caixa do projeto deverá ser considerada. Entretanto, muitas empresas não incorporam a mesma em suas análises ou, quando o fazem, a consideram homogênea ao longo de todo o período de avaliação (SOUZA, 2008).

No estudo realizado por Fensterseifer, Galesne e Ziegelmann (1987), foram encontrados índices relativamente baixos quanto à consideração da inflação diferenciada nos elementos constituintes do fluxo de caixa de um projeto. No ano de 1974, apenas $23 \%$ das empresas analisadas consideravam a inflação, enquanto no ano de 1985 houve um pequeno aumento nessa taxa, representando cerca de 35\% dos respondentes. 0 estudo de Fensterseifer e Saul (1993) apresentou índice de 58,9\% das empresas incorporando inflação nas análises econômicas, sendo destas $68,1 \%$ utilizando inflação homogênea e somente $31,9 \%$ considerando índices diferenciados conforme o componente do fluxo de caixa.

Uma pesquisa mais recente realizada por Arnold e Hatzopoulos (2000) apresentou novos índices para esse assunto, analisando empresas do Reino Unido. De acordo com os autores, cerca de 75\% das empresas acompanhadas faz o ajuste da inflação, seja ela constante para os elementos do fluxo de caixa, nesse caso usando a taxa real de desconto, ou diferenciada, fazendo, assim, uso da taxa nominal e realizando a correção do fluxo de caixa.

Este artigo teve como objetivo exemplificar as duas formas de incorporação da inflação em análises de investimentos: a homogênea e a heterogênea. Como resultado pode-se observar que essas duas formas de incorporação de inflação podem acarretar resultados bastante diferentes, na medida em que utilizam como base premissas também diferentes, conforme apresenta a Tabela 8 , consolidando os resultados obtidos durante a análise.

Este estudo demonstrou o impacto que a inflação diferenciada proporciona no resultado de um projeto. Observando a Tabela 8, percebe-se que, quando não considerada a inflação na avaliação de um projeto, provavelmente o resultado dessa avaliação estará distorcido, uma vez que deverá haver em períodos futuros a ocorrência de inflação. Avançando na análise da Tabela 8, percebe-se que a incorporação da inflação pode-se dar através de um único índice (homogênea) ou através de diferentes índices para cada componente do fluxo de caixa (heterogênea). No caso em estudo, a estratificação dos índices permitiu estimar um impacto bastante representativo no resultado final do projeto, tornando-o inviável caso as taxas de inflação estimadas venham a acontecer. Além disso, demonstrou-se que, caso a empresa tenha a possibilidade de depreciar seus investimentos em um período mais breve, estará aumentando a rentabilidade de seus projetos de investimentos, basicamente através da redução da descapitalização do valor a ser depreciado periodicamente.

Ainda no que tange à consideração da inflação heterogênea, dependendo dos índices de inflação estimada, ela poderá fazer com que projetos muito interessantes se tornem inviáveis (quando a inflação das despesas > inflação das receitas), ou que um projeto que já é considerado bom se torne mais interessante ainda (quando a inflação das receitas > inflação das despesas).

A consideração da inflação heterogênea de modo geral fornecerá um resultado mais aderente à realidade, uma vez que permitirá uma simulação mais próxima às reais variações monetárias que a empresa enfrentará. Entretanto, faz-se necessária uma discussão da relação benefício/custo da incorporação desse tipo de inflação, dado que em determinadas análises o tempo e precisão despendidos na montagem do fluxo de caixa não serão equivalentes ao impacto no resultado final da análise. Em casos como esse, a consideração da inflação de forma homogênea poderá ser satisfatória, pois as

Tabela 8. Resultados encontrados a partir das análises realizadas.

\begin{tabular}{|c|c|c|c|c|c|c|c|c|}
\hline \multirow{3}{*}{$\begin{array}{c}\text { Tipo de análise } \\
\text { Métodos de análise }\end{array}$} & \multicolumn{4}{|c|}{ Investimento com vida útil de 3 anos } & \multicolumn{4}{|c|}{ Investimento com vida útil de 2 anos } \\
\hline & \multicolumn{2}{|c|}{ Antes ir } & \multicolumn{2}{|c|}{ Depois ir } & \multicolumn{2}{|c|}{ Antes ir } & \multicolumn{2}{|c|}{ Depois ir } \\
\hline & VPL & TIR & VPL & TIR & VPL & TIR & VPL & TIR \\
\hline Sem inflação & $\mathrm{R} \$ 3.434,26$ & $30,64 \%$ & $\mathrm{R} \$ 1.743,20$ & $20,71 \%$ & - & - & - & - \\
\hline Inflação homogênea & $\mathrm{R} \$ 3.434,26$ & $30,64 \%$ & $\mathrm{R} \$ 1.495,54$ & $19,26 \%$ & $\mathrm{R} \$ 3.434,26$ & $30,64 \%$ & $\mathrm{R} \$ 1.661,07$ & $20,86 \%$ \\
\hline Inflação heterogênea & $\mathrm{R} \$ 1.029,22$ & $16,69 \%$ & $-R \$ 27,42$ & $9,82 \%$ & $\mathrm{R} \$ 1.029,22$ & $16,69 \%$ & $\mathrm{R} \$ 126,61$ & $10,88 \%$ \\
\hline
\end{tabular}


incorreções dos resultados serão compensados pela facilidade de operacionalização da análise.

Além disso, esses índices diferenciados de inflação foram considerados constantes por elemento ao longo do tempo. Uma análise ainda mais sofisticada exigiria que fossem considerados índices diferenciados por elementos e por período. Mais uma vez, a análise benefício/custo dessa sofisticação deverá ser constantemente avaliada.

No exemplo analisado ao longo do artigo, pode-se perceber que tanto no caso da inflação heterogênea quanto no caso da inflação homogênea a formulação utilizada é a mesma. Ou seja, pode-se concluir que a inflação homogênea é um caso particular da inflação heterogênea, onde, no primeiro caso, todos os elementos do fluxo de caixa são atualizados de acordo com um único índice.

Destaca-se que, em ambos os casos, a depreciação deve permanecer constante, isto é, sem atualização, para que possa ser transposto para a análise econômico-financeira o impacto da legislação que não permite a correção dos valores anuais a serem lançados como despesa. Pode-se considerar, assim, que a depreciação deverá ser atualizada com índice de $0 \%$ ao ano, enquanto os demais elementos do fluxo de caixa deverão ser atualizados conforme os índices que a empresa deverá definir, sejam eles idênticos para todos os elementos (inflação homogênea) ou diferenciados (inflação heterogênea).

Por fim, destaca-se que o impacto da inflação mostrou-se representativo, e que em países nos quais as taxas de inflação são mais elevadas é extremamente importante desenvolver a expertise para se trabalhar com taxas diferenciadas, ou seja, com a inflação heterogênea. Nesses casos, saber avaliar projetos considerando corretamente o impacto da inflação será um diferencial competitivo e poderá resultar na sobrevivência das empresas no mercado.

\section{Referências}

ALBERTON, A. et al. Seleção de Investimentos: aspectos e ferramentas relevantes na perspectiva dos gestores. In: ENCONTRO NACIONAL DE ENGENHARIA DE PRODUÇÃO, 24., 2004, Florianópolis. Anais... Florianópolis, 2004.

ARNOLD, G. C.; HATZOPOULOS, P. D. The TheoryPractice Gap in Capital Budgeting: evidence from the United Kingdom. Journal of Business Finance \& Accounting, v. 27, n. 5-6, p. 603, 2000. http://dx.doi. org/10.1111/1468-5957.00327

CASAROTTOFILHO, N.; KOPITTKE, B.H.Análise delnvestimentos: matemática financeira, engenharia econômica, tomada de decisão e estratégia empresarial. 9. ed. São Paulo: Atlas, 2000.

FARRAGHER, E. J.; KLEIMAN, R. T.; SAHU, A. P. Current Capital Investment Practices. The Engineering Economist, v. 44, n. 2, p. 137-150, 1999. http://dx.doi. org/10.1080/00137919908967513

FENSTERSEIFER, J. E.; GALESNE, A.; ZIEGELMANN, J. A utilização de Técnicas Analíticas nas Decisões de Investimentos de Capital das Grandes Empresas no Brasil. Revista de Administração de Empresas, v. 22, n. 4, p. $70,1987$.

FENSTERSEIFER, J. E.; SAUL, N. Investimentos de capital nas grandes empresas. Revista de Administração, v. 28, n. 3, p. 3-12, 1993.

GALESNE, A.; FENSTERSEIFER, J. E.; LAMB, R. Decisões de Investimentos da Empresa. São Paulo: Atlas, 1999.

KLIEMANN NETO, F. J. Apostila de Engenharia Econômica. Universidade Federal do Rio Grande do Sul. Disponível em: <http://www.producao.ufrgs.br/disciplinas.asp?cod_ turma $=87>$ Acesso em: dez. 2006.

SMART, S. B.; MEGGINSON, W. L.; GITMAN, L. J. Corporate Finance. Mason: South-Western, 2004.

SOUZA, J. S. Proposta de uma sistemática para análise multicriterial de investimentos. 2008. Dissertação (Mestrado em Engenharia de Produção)-Universidade Federal do Rio Grande Sul, Porto Alegre, 2008.

\section{The impact of inflation on project investment analysis}

\section{Abstract}

Investment analyses performed in a satisfactory way are essential for companies to apply capital resources in appropriate projects. To this end, the correct quantification of cash flow components, as well as the quantification of the depreciation, inflation and tributary impact of the income tax in economical-financial analysis is fundamental. Hence, the purpose of this study was to evaluate different forms of including inflation in investment analyses and discuss the depreciation role and its impacts. The article indicates two methods of analysis: homogeneous, where every component in the cash flow is updated by the same inflation index; and, heterogeneous, where each component is updated by a different inflation index. Results showed the impact of these two different concepts on the analysis, indicating the necessity of managers to focus on the issue of inflation during the assessment of their projects.

Keywords

Investment analysis. Depreciation. Inflation. 